\title{
Relação entre qualidade do sono e funções cognitivas em pacientes com doença de Parkinson
}

\author{
Altair Brito dos Santos ${ }^{1}$, Shirley L. Campos ${ }^{2}$, Sidarta Ribeiro ${ }^{3}$, Ludis Morales ${ }^{4}$, \\ Janneth Gonzalez ${ }^{4}$, Joabes dos SantosTrindade ${ }^{1}$, George E. Barreto ${ }^{4 冈}$
}

Relationship between quality of sleep and cognitive function in patients with Parkinson's disease

\begin{abstract}
Here, we describe the clinical characteristics of patients with Parkinson's disease, as well as study the relationship between sleep quality and cognitive functions. This is a descriptive-analytical cross-sectional study in which we evaluate episodes of depression and anxiety, as well as the state of general health, disease progression, sleepiness, sleep quality and cognitive functions of 24 patients with Parkinson's disease without dementia. We found a moderate inverse correlation between memory and sleep disturbances, and a moderate inverse correlation of memory and visuospatial function. We also observed other associations between subjective sleep quality and visuospatial function, sleep latency and the overall result of the SCOPA-COG, and between sleep duration and attention; all of these correlations were inverse and weak. This study suggests that mild cognitive deficits in the absence of dementia are associated with poor sleep quality.
\end{abstract}

Keywords: Parkinson's disease; sleep disorder; cognitive symptoms.

Edited by Alberto Acosta

1 Universidade Estadual do Sudoeste da Bahia, Jequié, BA, Brasil.

2 Departamento de Fisioterapia, Centro de Ciências da Saúde, Universidade Federal de Pernambuco, PE, Brasil.

3 Instituto do Cérebro, Natal, RN, Brasil.

4 Departamento de Nutrición y Bioquímica, Facultad de Ciencias, Pontificia Universidad Javeriana, Bogotá D.C., Colombia

Received: 12-07-2013 Accepted: 02-10-2013

Published on line: 21-10-2013

Citation: Brito dos Santos A, Campos S, Ribeiro S, Morales L, Gonzalez J, Trindade J, Barreto G (2013) Relação entre qualidade do sono e funções cognitivas em pacientes com doença de Parkinson. Universitas Scientiarum 18(3): 269-281 doi: 10.11144/ Javeriana. SC18-3. rqsf

Funding: N/A

Electronic supplementary material: N/A

SICI: 2027-1352(201309/12)18:3<269:REQDSEFCEPCDDP>2.0.TS;2-K

\section{Introdução}

A Doença de Parkinson é um distúrbio neurocomportamental definido clinicamente por seus sinais e sintomas motores (Friedman \& Millman 2008), apresentando também frequentes sintomas considerados não motores, como dor, disfunção olfativa, distúrbios do sono e comprometimento cognitivo (Aarsland et al. 2005, Chaudhuri et al. 2006). Além do seu papel como marcadores "pré-clínicos", essas características tornam-se mais proeminentes de acordo com a progressão da doença, aumentando o grau de deficiência dos portadores (Mehta et al. 2008).

Nas últimas décadas verificou-se um interesse crescente em distúrbios do sono na doença de Parkinson, que na visão de Arnulf et al. (2008) 
iniciou após a publicação de dois relatórios-chave. O primeiro relato foi feito por Schenck et al. (1996) que estudando Distúrbio do Comportamento do Sono REM idiopático observaram que num intervalo curto (menos de 3 anos) 37\% dos indivíduos estudados desenvolveram a doença de Parkinson. O segundo relato foi publicado por Frucht et al. (1999) onde observaram que alguns parkinsonianos experimentam ataques de sono quando tratados com agonistas dopaminérgicos.

Os Distúrbios do sono têm causas multifatoriais, alterações neuroquímicas e fisiopatológicas no Sistema Nervoso Central (SNC) (Goetz et al. 1986, Kostic et al. 1991, Starkstein et al. 1991, Menza \& Rosen 1995, Hwang et al. 1999), consequentes da doença que, juntamente com medicamentos antiparkinsonianos, interferem de forma significativa no sono (Hwang et al. 1999, Chahine et al. 2013). Os indivíduos frequentemente relatam dificuldade de iniciar ou manter o sono, parassonias, distúrbio do comportamento do sono REM, sonolência excessiva diurna, alterações nos sonhos e na atonia durante o sono (Hwang et al. 1999).

As alterações do sono estão presentes em 60\% a $90 \%$ dos portadores da Doença de Parkinson (Goetz et al. 1986, Kostic et al. 1991, Starkstein et al. 1991, Menza \& Rosen 1995, Trenkwalder 1998, Hwang et al. 1999, Aarsland et al. 2005, Gagnon et al. 2006, Chaudhuri et al. 2006, Arnulf et al. 2008, Friedman \& Millman 2008) dentre elas destaca-se o Distúrbio do Comportamento do Sono REM (DCR) que pode anteceder de 3 a 13 anos os sinais e sintomas motores (Gagnon et al. 2006), e está relacionado a um maior risco de disfunção cognitiva nos casos em que a doença não apresenta demência associada (Boeve et al. 2001, Boeve et al. 2003, Gagnon et al. 2006, Vendette et al. 2007). Estudos prévios indicam que os problemas no sono, além de diminuir consideravelmente a qualidade de vida, causam também déficits neuropsicológicos leves resultantes de prejuízos na memória, aprendizado e funções executivas (Boeve et al. 2001, Boeve et al. 2003, Ribeiro 2003, Gagnon et al. 2006, Vendette et al. 2007, Deschenes \& McCurry 2009). O sono, assim como também os sonhos (Ribeiro 2003), estão envolvidos em processos como a consolidação da memória e aprendizagem, principalmente em fenômenos como a aprendizagem para a resolução de problemas e melhora de habilidades procedural.

As evidências clínicas sugerem que distúrbios do sono especificamente o DCR pode ser um marcador pré-clínico para a demência na Doença de Parkinson, porém, são escassos os trabalhos que analisam a relação entre a qualidade do sono e os déficits cognitivos leves. Dessa maneira, esse estudo partiu do pressuposto de que a qualidade do sono pode também estar envolvida nas disfunções cognitivas. Sendo assim, essa pesquisa teve como objetivo descrever as características clínicas e demográficas de pacientes com Doença de Parkinson, e verificar a relação entre a qualidade subjetiva do sono e as funções cognitivas. Esse conhecimento é importante no desenvolvimento de estudos sobre novas perspectivas de avaliação, tratamento e prevenção da demência na Doença de Parkinson e auxiliar no direcionamento da elaboração de condutas mais eficazes para melhora da qualidade do sono.

\section{Materiais e métodos}

Trata-se de um estudo descritivo-analítico de corte transversal e abordagem quantitativa, realizado na cidade de Jequié, localizada no sudoeste da Bahia, Brasil. O estudo foi aprovado pelo Comitê de Ética em Pesquisa da Universidade Estadual do Sudoeste da Bahia - UESB sob protocolo n. 056/2010 e todos os pacientes leram e assinaram o termo de consentimento livre e esclarecido.

Amostra: A população deste estudo foi composta de pacientes com Doença de Parkinson da cidade de Jequié-BA (Brasil), cadastrados na $13^{\text {a }}$ Diretoria Regional de Saúde (DIRES) até o período de abril a maio de 2010. Dentre os 60 pacientes cadastrados, 24 pacientes preencheram os critérios de inclusão e exclusão. Foram incluídos os indivíduos com diagnóstico confirmado de Doença de Parkinson realizado por um médico neurologista, em uso contínuo de medicação dopaminérgica, de ambos os sexos e de qualquer faixa etária. Para impedir que variáveis conflitantes interferissem nos resultados de forma direta ou indireta, foram adotados como critério de exclusão: tratamento de alguma outra 
doença orgânica neurogênica ou psiquiátrica, presença de histórico de outras alterações neurológicas além da Doença de Parkinson, alterações psiquiátricas como alucinações e delírio, histórico neurocirúrgico e diagnóstico de demência associado à Doença de Parkinson ou pontuação insuficiente no Mini-Exame do Estado Mental (MEEM) (Folstein et al. 1975, Molloy et al. 1991, Zadikoff et al. 2007), tendo como ponto de corte os valores: 18 pontos para indivíduos analfabetos e 22 pontos para indivíduos com algum nível de instrução escolar.

Avaliação dos Pacientes: As entrevistas e testes foram realizados diretamente com os pacientes em uma ou mais sessões com uma duração de 60 a 90 minutos por um entrevistador alheio à pesquisa. Referidas entrevistas consistiram na execução de Anamnese para investigar o histórico de doença dos indivíduos e comorbidades associadas; realização de atividade física regular (sendo considerado válido o relato da realização de qualquer exercício físico com duração mínima de 30 min realizadas ao menos 3 vezes por semana); aplicação da Escala de Estágios de Incapacidade de Hoehn e Yahr (Degree of Disability Scale - HY) (Hoehn \& Yahr 1967) para avaliação do estado geral do paciente e aplicação da Escala Unificada de Avaliação da Doença de Parkinson (Unified Parkinson's Disease Rating Scale - UDPRS) (Fahn et al. 1987) para avaliação da progressão da doença. A UPDRS é uma escala composta por 4 domínios, em que a maioria dos itens são pontuados de zero (normal) a quatro (prejudicado): UPDRS-I consiste em quatro itens que avalia o estado mental, emocional e comportamental; UPDRS-II é composto de treze itens descrevendo a capacidade de executar as atividades de vida diária; UPDRS-III é uma classificação dos sinais motores da doença e UPDRS-IV avalia as taxas de complicações da terapia, incluindo questões relativas à duração/ intensidade das discinesias e flutuações motoras, juntamente com três itens relativos à anorexia, distúrbio do sono e hipotensão ortostática.

A presença de depressão e ansiedade também foram investigadas por tratarem-se de alterações psicológicas comuns na Doença de Parkinson e de grande impacto nas alterações do sono. Para tal, aplicaram-se as versões brasileiras do Inventário de Depressão de Beck - BDI (Beck et al. 1961, Rosa et al. 1986, Beck \& Steer 1993) e do Inventário de Ansiedade de Beck - BAI (Cunha 2001). A disfunção do sono foi avaliada por meio da Escala de Sonolência de Epworth, a qual mede e classifica a sonolência (Hoddes et al. 1973, Marinus et al. 2003). A escala avalia a propensão do paciente para adormecer em oito situações diferentes em uma escala de 0-3. Uma pontuação maior que 10 é sugestiva de sonolência excessiva diurna, e maior que 15 é sugestiva de sonolência patológica.

Para avaliar a qualidade subjetiva do sono e distúrbios associados, aplicou-se o questionário do Índice de Qualidade do Sono de Pittsburgh (IQSP). O IQSP foi desenvolvido por Buysse et al. (1989) e validado no Brasil por Ceolim (1999). É composto por 19 itens relacionados aos hábitos de sono do mês anterior ao que o indivíduo se encontra, sendo a combinação de 7 componentes: (01) Qualidade subjetiva do sono, (02) latência do sono (tempo necessário para iniciá-lo), (03) duração do sono (horas de sono por noite), (04) eficiência habitual do sono (tempo total de sono dividido pelo tempo na cama), (05) distúrbios do sono (por exemplo, acordar no meio da noite), (06) uso de medicação para dormir e (07) disfunção diurna (ter dificuldade para ficar acordado). Cada componente recebe uma pontuação que varia de 0 a 3. Os escores de todos os componentes são somados a fim de se obter um valor global com variação de 0 a 21 pontos. Escores globais maiores que 5 indicam qualidade ruim do sono e escores maiores que 10 , a presença de distúrbios do sono associados.

A avaliação da memória e funções cognitivas dos pacientes foi realizada por meio do Scales for Outcomes in Parkinson's disease-Cognition (SCOPACOG) (Marinus et al. 2003) que consiste num instrumento curto, confiável, validado e sensível ao déficit cognitivo específico para Doença de Parkinson, com versão em português (CarodArtal et al. 2008). É composto por 10 itens, tendo os seguintes domínios: memória, atenção, funções executivas e as funções visuoespaciais. O domínio "memória" avalia as memórias visual, verbal, imediata e recordação tardia. Duas 
tarefas são incluídas no domínio “atenção", a contagem regressiva de três em três. $\mathrm{O}$ domínio "função executiva" avalia a fluência semântica e o planejamento motor enquanto que a "função visuoespacial" é avaliada em uma área de montagem com uma figura-tarefa. O SCOPA-COG possui um escore total máximo de 43 pontos, sendo que pontos mais altos refletem melhor desempenho.

Estatística: Os dados foram analisados utilizando o programa Statistical Packeage for Social Sciences (SPSS), versão 18.0 for Windows. As variáveis categóricas, gênero, idade, estado geral estado civil, atividade física regular, dores musculoesqueléticas, depressão, ansiedade, estado geral e sonolência diurna foram analisadas quanto à distribuição de frequência absoluta e relativa. As variáveis quantitativas funções cognitivas (memória, atenção, funções executivas e função visuoespacial), qualidade do sono e seus componentes, pontuação dos domínios e global do UPDRS foram apresentadas seus valores de média, percentis, desvio padrão, amplitude, valores máximos e mínimos. A qualidade do sono foi tratada de forma categórica e de forma quantitativa.

O teste Smirnov-Kolmogorov foi aplicado para avaliar a distribuição dos dados, a qual se mostrou não-normal. Sendo assim, foi usada a correlação de Spearman (rho) para testar a relação entre a qualidade subjetiva do sono e as funções cognitivas por meio de: (i) escore da qualidade do sono versus escore do UPDRS-I (referente ao estado mental, emocional e comportamental) e (ii) escore da qualidade do sono (e seus componentes) versus escore do SCOPA-COG (e seus domínios). Para todas as correlações considerou-se um nível de significância para p-valor $<0,05$.

\section{Resultados}

Quanto às características clínicas e demográficas dos pacientes estudados, verificou-se que 70,8\% foram do sexo masculino, $50 \%$ dos indivíduos possuíam idade menor que 65 anos, com média de $66,8+/$ - 9,6 anos e que a maioria (91,7\%) possui vida conjugal. Todos utilizavam pelo menos um medicamento antiparkinsoniano (levodopa, pramipexole, amantadina, biperideno) e a dose diária equivalente de levodopa teve um valor médio de $783.9+/-417.7$ (Tabela 1). Encontravam-se classificados no estágio III da escala de Hoehn e Yahr 41,7\% dos pacientes, e os demais distribuídos igualitariamente nos estágios II e IV (ambos 25\%). O maior percentual desses indivíduos $(91,7 \%)$ relatou sentir alguma dor musculoesquelética (Tabela 1).

Tabela 1. Distribuição das frequências absolutas e relativas das características clínicas e demográficas dos indivíduos investigados, Jequié 2010.

\begin{tabular}{|c|c|c|c|}
\hline Características Gerais & & $\mathbf{N}$ & $\%$ \\
\hline \multirow{3}{*}{ Gênero } & Feminino & 7 & 29,2 \\
\hline & Masculino & 17 & 70,8 \\
\hline & Total & 24 & \\
\hline \multirow{5}{*}{ Idade } & $<65$ anos & 12 & 50,0 \\
\hline & $=65$ a 69 anos & 2 & 8,3 \\
\hline & 70 a 74 anos & 4 & 16,7 \\
\hline & 75 a 79 anos & 6 & 25,0 \\
\hline & Total & 24 & \\
\hline \multirow{5}{*}{$\begin{array}{l}\text { Estadiamento } \\
\text { da Doença }\end{array}$} & Estágio 1 & 2 & 8,3 \\
\hline & Estágio 2 & 6 & 25,0 \\
\hline & Estágio 3 & 10 & 41,7 \\
\hline & Estágio 4 & 6 & 25,0 \\
\hline & Total & 24 & \\
\hline \multirow{3}{*}{ Estado Civil } & com vida conjugal & 22 & 91,7 \\
\hline & sem vida conjugal & 2 & 8,3 \\
\hline & Total & 24 & \\
\hline \multirow{4}{*}{$\begin{array}{l}\text { Atividade Física } \\
\text { Regular }\end{array}$} & Sim & 12 & 50,0 \\
\hline & Não & 11 & 45,8 \\
\hline & Sem resposta & 1 & 4,2 \\
\hline & Total & 24 & \\
\hline \multirow{3}{*}{$\begin{array}{l}\text { Dores } \\
\text { Musculoesqueléticas }\end{array}$} & Sim & 22 & 91,7 \\
\hline & Sem resposta & 2 & 8,3 \\
\hline & Total & 24 & 100,0 \\
\hline \multirow{5}{*}{$\begin{array}{l}\text { Beck Inventário } \\
\text { de Depressão }\end{array}$} & Mínimo: 0 a 9 pontos & 7 & 29,2 \\
\hline & Leve: 10 a 17 pontos & 5 & 20,8 \\
\hline & Moderada: 18 a 24 pontos & 7 & 29,2 \\
\hline & Grave: $>24$ pontos & 5 & 20,8 \\
\hline & Total & 24 & \\
\hline \multirow{5}{*}{$\begin{array}{l}\text { Beck Inventário } \\
\text { de Ansiedade }\end{array}$} & Mínimo: 0 a 10 pontos & 6 & 25,0 \\
\hline & Leve: 11 a 19 pontos & 7 & 29,2 \\
\hline & Moderado: 20 a 30 pontos & 5 & 20,8 \\
\hline & Grave: $>30$ pontos & 6 & 25,0 \\
\hline & Total & 24 & \\
\hline
\end{tabular}


Tabela 2. Distribuição das frequências absolutas e relativas dos resultados do Índice de Qualidade de Sono de Pittsburgh (ISQP) e Escala de Sonolência de Epworth dos indivíduos investigados, Jequié 2010.

\begin{tabular}{llll}
\hline Respostas da avaliação do sono & & N & $\%$ \\
\hline \multirow{3}{*}{ Classificação de Pittsburgh } & Boa qualidade de sono: 0 a 4 pontos & 6 & 25,0 \\
& Qualidade ruim de sono: 5 a 10 pontos & 13 & 54,2 \\
& Distúrbio do sono: >10 pontos & 5 & 20,8 \\
& Total & 24 & \\
& Sonolência diurna normal: 0 a 9 pontos & 12 & 50,0 \\
& Sonolência diurna excessiva: 10 a 15 pontos & 2 & 8,3 \\
Classificação da Sonolência Diurna & Sonolência diurna patológica: >15 pontos & 2 & 8,3 \\
& Total & 24 & \\
\hline
\end{tabular}

No que se refere à prática de exercícios físicos houve uma discreta predominância quanto ao percentual de indivíduos que praticam $(50 \%)$ e não praticam $(45,8 \%)$ atividade física regular (Tabela 1). $\mathrm{Na}$ avaliação de episódios de depressão e ansiedade, os maiores percentuais foram observados para indivíduos com depressão mínima e moderada (ambas com 29,0\%) e ansiedade leve (29,2\%) (Tabela 1).

A avaliação do sono foi dividida em qualidade do sono e sonolência diurna, apresentadas na tabela 2 por meio das escalas do Índice de Qualidade do Sono de Pittsburgh e Escala de Epworth. Os resultados mostraram uma maior frequência de pacientes com uma qualidade ruim de sono e com distúrbios associados somando 75\%. Sendo que $54,2 \%$ tem apenas qualidade ruim do sono e $20,8 \%$ apresentam qualidade ruim sono com distúrbios associados (Tabela 2). A sonolência excessiva foi observada em $50 \%$ dos pacientes, sendo que um percentual de 8,3\% apresentou sonolência diurna considerada patológica, resultado esperado como consequência de uma elevada frequência de paciente com qualidade de sono noturno ruim (Tabela 2). Na tabela 3 encontra-se outra análise dos resultados do IQSP, e verificaram-se resultados similares, de média e percentil, com valores relativamente altos $(7,08+/-3,84$ pontos $)$, $(\mathrm{p} 25=5,75, \mathrm{p} 50=6,75, \mathrm{p} 75=9,00)$ denotando que a maior parte dos pacientes atingiram pontuação classificada como qualidade ruim do sono.

$\mathrm{Na}$ análise detalhada (Tabela 3) dos componentes da qualidade do sono, verificou-se que os componentes mais prejudicados foram, em ordem crescente, a qualidade percebida do sono, disfunção diurna (ter dificuldade para ficar acordado), latência do sono (tempo necessário para iniciá-lo) e alterações do sono (por exemplo, acordar no meio da noite) (Tabela 3). Nos resultados observaram-se a presença de fatores que contribuem de formas direta e indireta para redução da qualidade do sono noturno, e consequentemente aumento da sonolência diurna, como por exemplo, quase todos os pacientes são acometidos de alguma dor musculoesquelética, todos os pacientes utilizam medicamentos antiparkinsonianos e seus efeitos adversos também interferem no sono.

Os pacientes apresentaram média global no UPDRS de 40,17 +/- 17,22 pontos, tendo o valor mínimo de 11 e o máximo de 97 pontos. Devese ressaltar que, a mediana foi próxima a média ( $\mathrm{P} 50=41,75$ pontos) e que $75 \%$ dos pacientes obtiveram pontuações até 48 pontos. Na parte I do UPDRS, a mais interessante para os objetivos 
Tabela 3. Resultados dos domínios e das pontuações globais do UPDRS, ISQP, e SCOPA-COG, Jequié 2010. DP - Desvio-padrão; P 25 - percentil 25; P 50 - percentil 50; P75 - percentil 75; Mín. - Mínimo; Máx - Máximo.

\begin{tabular}{|c|c|c|c|c|c|c|c|c|}
\hline Pontuação Global e de Domínios & $\mathbf{N}$ & Média & DP & Mín & Máx & P25 & P50 & P75 \\
\hline UPDRS & 24 & 40,17 & 17,22 & 11 & 97 & 30,75 & 41,75 & 48,00 \\
\hline Parte I: Estado Mental, Emocional e Comportamento & 24 & 3,21 & 2,02 & $\mathbf{0}$ & 7 & 2,00 & 3,00 & 4,00 \\
\hline Parte II: Atividade de Vida Diária & 24 & 13,50 & 6,17 & 4 & 31 & 10,00 & 12,50 & 17,00 \\
\hline Parte III: Exame Motor & 24 & 16,58 & 8,41 & 3 & 43 & 12,00 & 16,00 & 20,25 \\
\hline Parte IV: Complicações na Terapia & 24 & 6,87 & 3,87 & 1 & 16 & 4,75 & 6,00 & 9,00 \\
\hline Qualidade Percebida do Sono & 24 & 1,08 & 0,50 & $\mathbf{0}$ & 3 & 1,00 & 1,00 & 1,00 \\
\hline Latência do Sono & 24 & 1,25 & 1,11 & $\mathbf{0}$ & 3 & 1,00 & 1,00 & 1,00 \\
\hline Duração do Sono & 24 & 0,88 & 1,15 & $\mathbf{0}$ & 3 & 1,00 & 1,00 & 1,00 \\
\hline Eficiência Habitual do Sono & 24 & 0,25 & 0,73 & 0 & 3 & 0,00 & 0,00 & 0,00 \\
\hline Alterações do Sono & 24 & 1,33 & 0,70 & 0 & 3 & 1,00 & 1,00 & 2,00 \\
\hline Uso de Medicação & 24 & 0,88 & 1,39 & $\mathbf{0}$ & 3 & 0,00 & 0,00 & 3,00 \\
\hline Disfunção Diurna & 24 & 1,21 & 0,97 & $\mathbf{0}$ & 3 & 1,00 & 2,00 & 2,00 \\
\hline Pittsburgh & 24 & 7,08 & 3,84 & 3 & 16 & 5,75 & 6,75 & 9,00 \\
\hline Memoria e Aprendizado & 24 & 7,25 & 2,41 & 3 & 13 & 6,00 & 7,00 & 8,00 \\
\hline Atenção & 24 & 2,62 & 1,27 & 0 & 4 & 2,00 & 2,00 & 4,00 \\
\hline Funções Executivas & 24 & 5,20 & 2,04 & 2 & 8 & 4,00 & 4,50 & 7,00 \\
\hline Função Visuo-espacial & 24 & 2,54 & 0,83 & 1 & 4 & 2,00 & 3,00 & 3,00 \\
\hline SCOPA-COG & 24 & 16,95 & 4,92 & 7 & 23 & 14,75 & 19,50 & 22,00 \\
\hline
\end{tabular}

desse estudo, os pacientes apresentaram uma média relativamente baixa 3,21 +/- 2,02 pontos, a qual também foi próxima à mediana $(3,00$ pontos), indicando um bom estado mental dos pacientes (Tabela 3). Explica-se esse resultado pelo fato, de terem sido excluídos os pacientes que apresentavam quadro característico de demência, ou pontuação insuficiente no MEEM, também é verificado e que não houve interferência de idades avançadas, e nem maior frequência de paciente no estágio IV da escala de Hoehn e Yahr.

Os resultados da avaliação cognitiva dos pacientes mostraram uma média global relativamente baixa (16,95 +/- 4,92 pontos), sendo o P50 de 19,50 e o P75 de 22 pontos. Os domínios mais prejudicados foram memória com média 7,25 pontos $(+/-2,41)$ e funções executivas com média de 5,20 pontos (+/- 2,04) (Tabela 3). A maior frequência de idades avançadas, poderia ser considerada um fator de interferência neste resultado, visto que com o avançar da idade há uma queda das funções cognitivas, porém houve uma maior frequência de idades menos avançadas.

Nos resultados deste estudo foram encontrados um percentual elevado de pacientes com qualidade ruim do sono e a média geral do teste de funções cognitivas foi baixa, o que sugeriu uma relação entre eles, sendo assim foram aplicados os testes 
estatísticos inferenciais para confirmação. $\mathrm{Na}$ análise da correlação, entre o sono e alterações cognitivas dos pacientes, verificou-se a relação da qualidade do sono, e seus componentes, com a parte I do UPDRS, (que avalia o estado mental, emocional e comportamental, geral dos indivíduos), e foi encontrada correlação positiva e significativa, considerada moderada com o componente alterações do sono (Correlação de Spearman, $R h o=0,512, p=0,010)$, denotando que quanto mais alterações no sono pior o estado mental, emocional e comportamental geral dos pacientes (Tabela 4).

A relação entre o UPDRS (parte I) e a disfunção diurna foi positiva, e considerada fraca (Correlação de Spearman, $R h o=0,419, p=0,041)$, denotando que quanto maior a disfunção diurna pior o estado mental, emocional e comportamental geral (Tabela 4). Foi verificada também a relação entre as médias global do ISQP e do UPDRS (parte I) tendo com o resultado uma correlação positiva, fraca (Correlação de Spearman, Rho $=0,418$, $p=0,042)$, mostrando que quanto pior a qualidade do sono, também pior o estado mental, emocional, comportamental geral dos pacientes (Tabela 4).
As relações entre o sono e as funções cognitivas foram verificadas utilizando a pontuação global e as pontuações dos componentes do ISQP versus a pontuação global e domínios do SCOPA-COG. Analisando os resultados do teste de memória tardia e a as alterações do sono, encontrou-se correlação do tipo inversa e moderada (Correlação de Spearman, $R h o=-0,520, p=0,009)$, denotando que quanto mais alterações do sono pior a memória tardia dos pacientes. O componente latência do sono esteve também correlação inversa e moderada, com a memória (Correlação de Spearman, Rho= - 0,512, $p=0,011)$, mostrando que quanto mais tempo se demorava para iniciar o sono piores eram os desempenhos nas tarefas de memória. E também correlacionada de forma inversa e moderada com a função visuoespacial (Correlação de Spearman, $R h o=-0,509, p=0,011), \log$ o quanto mais tempo o paciente demorava em iniciar o sono pior foi seu desempenho na tarefa visuoespacial.

Outras relações foram observadas tais como entre os resultados do componente qualidade subjetiva do sono e do domínio função visuoespacial (Correlação de Spearman, $R h o=$ -0,402, $p=0,050)$, entre o componente latência

Tabela 4. Resultados das correlações entre qualidade do sono e cognição.

\begin{tabular}{|lcc|}
\hline Correlações & Rho & P \\
\hline UPDRS - Parte I vs Alteração do sono & 0,512 & 0,010 \\
\hline UPDRS - Parte I vs Difusão diurna & 0,419 & 0,041 \\
\hline UPDRS - Parte I vs Pittsburgh & 0,418 & 0,042 \\
\hline Qualidade Percebida do Sono vs Funções visuo-espaciais & $-0,402$ & 0,050 \\
\hline Latência do Sono vs Memória & $-0,512$ & 0,011 \\
\hline Latência do Sono vs Função Visuo Espacial & $-0,509$ & 0,011 \\
\hline Latência do Sono vs Scopa Soma & $-0,451$ & 0,027 \\
\hline Duração do Sono vs Atenção & $-0,439$ & 0,032 \\
\hline Alterações do sono vs Memória Tardia & $-0,520$ & 0,009 \\
\hline
\end{tabular}


do sono e o resultado global do SCOPACOG (Correlação de Spearman, Rho = - 0,451, $p=0,027$, e entre o componente duração do sono e o domínio atenção (Correlação de Spearman, $R h o=-0,439, p=0,032)$. Todas foram correlações inversas, e também de caráter fraco (Tabela 4). Sendo assim quanto pior a qualidade percebida do sono pelo paciente, pior também eram os seu resultado na tarefa visuoespacial. Quanto mais tempo os pacientes demoravam em iniciar o sono noturno, pior era seu desempenho cognitivo geral, e ainda quanto menor a duração do sono menor era o desempenho nas tarefas de atenção.

Testes de correlação foram realizados, entre características clinicas (como dor musculoesquelética, atividade física) e o ISQP e seus componentes, Escala de Sonolência de Epworth e SCOPA-Cog e seus domínios, além de outros, porém encontrou-se um nível de significância com $\mathrm{P}>0,05$, tornando o resultado impróprio para esse estudo. Por outro lado, não deve ser descartado sua importância para outros estudos, que poderá obter resultados significativos utilizando amostra com um maior número de pacientes.

\section{Discussão}

Esse estudo teve como objetivo descrever as características clínicas e demográficas de pacientes com Doença de Parkinson, e verificar a relação entre a qualidade subjetiva do sono e funções cognitivas. $\mathrm{E}$ os resultados sugerem que a qualidade do sono pode estar relacionada aos déficits cognitivos de intensidade leve na Doença de Parkinson. Nesse estudo foram observados prejuízos nas funções cognitivas (memória, funções executivas, função visuoespacial e atenção) dos pacientes, corroborando com observações prévias que relatam que esses comprometimentos estão presentes nos diversos estágios da Doença de Parkinson (DP), e principalmente na memória (Janvin et al. 2003), nas funções executivas (Wright et al. 1990, Green et al. 2002, Foltynie et al. 2004, Muslimovic et al. 2005, Iranzo et al. 2006), na função visuoespacial (Levin et al. 1991, Green et al. 2002, Foltynie et al. 2004, Muslimovic et al. 2005) e atenção (Wright et al. 1990, Green et al. 2002, Foltynie et al. 2004, Muslimovic et al. 2005).
E concomitantemente foram encontrados um maior percentual de pacientes que apresentavam qualidade ruim do sono e sonolência diurna excessiva assim como em estudos anteriores (Lavie et al. 1980, Van Hilten et al. 1994, Arnulf et al. 2002, Brodsky et al. 2003, Razmy et al. 2004, Stevens et al. 2004, Gjerstad et al. 2006, Porter et al. 2008, Zhang et al. 2013).

A idade avançada poderia ser considerada como um dos principais fatores para explicar os déficits cognitivos, no entanto, uma grande porcentagem da amostra era de pacientes com idade inferior aos 65 anos. A distribuição de gênero, média de idade e uso de terapia medicamentosa (dose diária de levodopa) dos pacientes estudados são semelhantes à descrição de outros autores (Elena et al. 2008, Massicotte-Marquez et al. 2008, Marion et al. 2008, Colman et al. 2009, Rossi et al. 2009). Também foram encontradas similaridades quanto as frequências observadas para o estágio da doença (Elena et al. 2008, Marion et al. 2008, Colman et al. 2009, Rossi et al. 2009), prevalência de episódios de depressão (Elena et al. 2008, Marion et al. 2008, Colman et al. 2009, Rossi et al. 2009), ansiedade (Troster et al. 1995, Richard et al. 1996) e média global do UPDRS (Elena et al. 2008, Marion et al. 2008, Colman et al. 2009, Rossi et al. 2009). A partir das correlações encontradas nesse estudo, entende-se que com a redução da qualidade do sono (denotando um aumento das pontuações no ISQP), os pacientes apresentaram um menor desempenho cognitivo (denotando redução das pontuações no SCOPA-COG), e o aumento da latência do sono está relacionado com um pior desempenho de memória e um declínio da função visuoespacial. Adicionalmente, a redução na duração do sono também está relacionado com um pior desempenho da atenção, e quanto maiores às alterações do sono, pior foi o desempenho de memória tardia, sugerindo, portanto, que a qualidade do sono pode influenciar no funcionamento cognitivo de pacientes com Doença de Parkinson.

Estudos que investigaram a relação entre a qualidade do sono e os transtornos cognitivos na Doença de Parkinson, são escassos, porem em um estudo similar, Stavitsky et al. (2012), encontraram 
uma relação ente a pior qualidade do sono com as funções cognitivas e seus domínios, tais como com a atenção o que também corrobora com esse estudo, e com as funções executivas, o que não foi visto neste estudo, dentre outros motivos podemos colocar devido algumas limitações metodológicas tais como o tamanho da população estudada.

Estudos prévios sugerem que a presença de alterações do sono como Distúrbio do Comportamento do Sono REM (DCR), nos pacientes com DP, representa um fator de risco para o desenvolvimento de déficits cognitivos leves e posterior progressão para demência, que é uma característica marcante na doença (Vendette et al. 2007, Massicotte-Marquez et al. 2008, Perfetti et al. 2010). A partir de um estudo longitudinal de Aarsland et al. (2005) observou-se que a maioria dos pacientes com DP desenvolveram demência durante um período de 8 anos, e em um outro estudo realizado por Javin et al. (2006) descreveu que durante um período de 4 anos, $82 \%$ dos pacientes desenvolveram a demência, dentre estes $62 \%$ apresentavam inicialmente déficits cognitivos leves e $20 \%$ não apresentavam alterações cognitivas. Vários fatores como idade avançada, idade de início dos sintomas motores, a ocorrência precoce de confusão mental relacionada a complicações na terapia medicamentosa, psicose, depressão, sintomas motores (graves ou atípicos), também têm sido associados a um maior risco para desenvolver a demência (Aarsland et al. 2005).

Estudos de imaginologia cerebral realizados em pacientes com Doença de Parkinson e déficits cognitivos e em pacientes com DCR mostraram alterações neurais em comum nos vários núcleos do tronco cerebral (substância negra, núcleo do pedúnculo pontino, locus coeruleus, núcleo complexo subcoeruleus, núcleos da rafe) e anomalias em seus neurotransmissores dopaminérgicos, colinérgicos, noradrenérgicos e serotoninérgico (Braak et al. 2005, Vendette et al. 2007, Rossi et al. 2009). Todas estas estruturas apresentam projeções difusas para o córtex cerebral, e perturbações destas redes neurais podem explicar a presença de déficits cognitivos em pacientes com Doença de Parkinson e distúrbios do sono.
Foram observadas algumas limitações metodológicas neste estudo, tais como as avaliações do sono não foram confirmadas com a polissonografia, baseando-se apenas na entrevista clínica com ISQP e Escala de Sonolência de Epworth, que já tinham demonstrado ter alta sensibilidade e especificidade para quantificar a qualidade do sono na Doença de Parkinson (Buysse et al. 1989, Ceolim 1999). A identificação dos distúrbios do sono não foi verificada, tais como síndrome da apneia obstrutiva (uma situação que deve ser evitada por estar relacionada com a disfunção do lobo frontal) e o distúrbio do comportamento do sono REM, podendo alterar os resultados. Além disso, não houve grupo controle e os resultados dos testes cognitivos foram comparados apenas aos dados publicados na literatura. Verifica-se também que a ocorrência de multicolinearidade não foi analisada. Para resultados mais apurados sugere-se a realização de estudos com uma amostra maior com a execução de testes de regressão múltipla.

Existem várias consequências práticas desses resultados sobre a associação entre a qualidade do sono e funções cognitivas em pacientes com Doença de Parkinson. A avaliação subjetiva dessas variáveis mediante a aplicação desses questionários, abre a possibilidade para futuros estudos controlados em laboratórios do sono e com controle de vídeo-polissonografia, além da implementação de testes mais específicos para a confirmação de comprometimento cognitivo leve. Esta hipótese, caso confirmada, seria de grande importância na triagem clínica, já que a qualidade ruim do sono se tornaria também um marcador pré-clínico para os riscos de demência.

\section{Conclusão}

No que se refere às características clínicas e demográficas dos pacientes com Doença de Parkinson em estudo, conclui-se que eles apresentaram prevalência de alterações psicológicas, como episódios de depressão e ansiedade, alterações motoras, alterações do sono, como sonolência diurna excessiva, qualidade ruim do sono e distúrbios do sono associados. As funções cognitivas 
também encontram-se alteradas, tais como déficits da memória, atenção, funções executivas e função visuoespacial. Esse estudo sugere que os déficits cognitivos leves, na ausência de demência, estão relacionados à qualidade ruim do sono.

\section{Agradecimentos}

Agradecemos aos colegas que contribuíram de forma indireta para esta pesquisa, a Universidade Estadual do Sudoeste da Bahia - UESB pelo apoio e autorização pelo comitê de ética em pesquisa sob o protocolo $\mathrm{n}$. 056/2010, e aos pacientes participantes da pesquisa. Fonte de financiamento: PUJ \#004509, 004580 y 004327.

\section{Conflitos de interesse}

Os autores declararam não haver conflitos de interesse.

\section{Referências}

Aarsland D, Zaccai J, Brayne C (2005) A systematic review of prevalence studies of dementia in Parkinson's disease. Movement Disorder 20: 12551263. doi: 10.1002/mds.20527

Arnulf I, Konofal E, Merino-Andreu M, Houeto JL, Mesnage V, et al. (2002) Parkinson's disease and sleepiness: an integral part of PD. Neurology 58 (7): 1019-1024. doi: 10.1212/WNL.58.7.1019

Arnulf I, Leu S, Oudiette D (2008) Abnormal sleep and sleepiness in Parkinson's disease. Current Opinion in Neurology 21: 472-477 doi: 10.1097/ WCO.0b013e328305044d

Beck AT, Steer RA (1993) Beck Depression Inventory. Manual. Psychology Corporation, San Antonio, USA.

Beck AT, Ward CH, Mendelson M, Mock J, Erbaugh J (1961) An inventory for measuring depression. Archives of General Psychiatry 4: 561-571

Boeve BF, Silber MH, Ferman TJ, Lucas JA, Parisi JE (2001) Association of REM sleep behavior disorder and neurodegenerative disease may reflect an underlying synucleinopathy. Movement Disorder 16: 622-630. doi: 10.1002/mds.1120

Boeve BF, Silber MH, Parisi JE, Dickson DW, Ferman TJ, et al. (2003) Synucleinopathy pathology and REM sleep behavior disorder plus dementia or parkinsonism. Neurology 6: 40-45. doi: 10.1212/01. WNL.0000073619.94467.B0

Braak H, Ru“b U, Jansen Steur EN, Del Tredici K, de Vos RA (2005) Cognitive status correlates with neuropathologic stage in Parkinson disease. Neurology 64 (8): 1404-10. doi: 10.1212/01. WNL.0000158422.41380.82

Brodsky MA, Godbold J, Roth T, Olanow CW (2003) Sleepiness in Parkinson's disease: a controlled study. Movement Disorder 18 (6): 668-672. doi: 10.1002/ mds.10429

Buysse DJ, Reynolds CF 3rd, Monk TH, Berman SR, Kupfer DJ (1989) The Pittsburgh Sleep Quality Index: a new instrument for psychiatric practice and research. Journal of Psychiatry Research 28 (2): 193-213. http://dx.doi.org/10.1016/0165-1781(89)90047-4

Carod-Artal FJ, Martínez-Martin P, Kummer W, Ribeiro LS, et al. (2008) Psychometric Attributes of the SCOPA-COG Brazilian Version. Movement Disorder 23 (1): 81-87. doi: 10.1002/mds.21769

Ceolim MF (1999) Padrões de atividades e de fragmentação do sono em pessoas idosas. Tese de Doutorado. Escola de Enfermagem de Ribeirão Preto/ Universidade de São Paulo, Brasil

Chahine LM, Daley J, Horn S, Duda JE, Colcher A, et al. (2013) Association between dopaminergic medications and nocturnal sleep in earlystage Parkinson's disease. Parkinsonism and Related Disorder 19 (10): 859-63. doi: 10.1016/j. parkreldis.2013.05.009

Chaudhuri KR, Healy DG, Schapira AH (2006) Nonmotor symptoms of Parkinson's disease: diagnosis and management. The Lancet Neurology 5: 235-245. http://dx.doi.org/10.1016/S1474-4422(06)703738

Colman KSF, Koerts J, Beilen M, Leenders VKL, Post WJ, Bastiaanse R (2009) The impact of executive functions on verb production in patients with Parkinson's disease. Cortex 45: 930-942. http:// dx.doi.org/10.1016/j.cortex.2008.12.010

Cunha JA (2001) Manual da versão em português das Escalas Beck. Casa do Psicólogo, São Paulo, Brasil.

Deschenes CL, McCurry SM (2009) Current Treatments for Sleep Disturbances in Individuals With Dementia. Current Psychiatry Reports 11 (1): 20-6.

Elena SE, Pacchetti C, Zangaglia R, Pasotti C, Manni R et al. (2008) REM Behavior Disorder, Hallucinations and Cognitive Impairment in Parkinson's Disease: A Two-Year Follow Up. Movement Disorder 23 (10): 1441-1445. doi: 10.1002/mds.22126

Fahn S, Elton RL, and members of the UPDRS Development Committee (1987) Unified Parkinson's Disease Rating Scale. In: Fahn S, Marsden CD, Calne D, Goldstein M. Recent developments in Parkinson's disease. Macmillan Healthcare Information, Florham Park NJ, USA, pp 153-63 
Folstein MF, Folstein SE, McHugh PR (1975) "MiniMental State": a practical method for grading the cognitive state of patients for the clinician. Journal of Psychiatry Research 12: 196-8. http://dx.doi. org/10.1016/0022-3956(75)90026-6

Foltynie T, Brayne CE, Robbins TW, Barker RA (2004) The cognitive ability of an incident cohort of Parkinson's patients in the UK. The Campaign study. Brain 127: 550-560. doi: 10.1093/brain/awh067

Friedman JH, Millman RP (2008) Sleep Disturbances and Parkinson 's Disease. CNS Spectrums 13: 3 (Supp 4): $12-17$

Frucht S, Rogers JD, Greene PE, Gordon MF, Fahn S (1999) Falling asleep at the wheel: motor vehicle mishaps in persons taking pramipexole and ropinirole. Neurology 52: 1908-1910. http://dx.doi. org/10.1212/WNL.52.9.1908

Gagnon JF, Postuma MR, Mazza S, Doyon J, Montplaisir J (2006) Rapid-eye-movement sleep behaviour disorder and neurodegenerative diseases. The Lancet Neurology 5: 424-432. http://dx.doi. org/10.1016/S1474-4422(06)70441-0

Gjerstad MD, Alves G, Wentzel-Larsen T, Aarsland D, Larsen JP (2006) Excessive daytime sleepiness in Parkinson disease: is it the drugs or the disease? Neurology 67 (5): 853-858

Goetz CG, Wilson RS, Tanner CM, Garron DC (1986) Relationships among pain, depression, and sleep alterations in Parkinson's disease. In: Yahr MD, Bergmann KJ, editors. Advances in Neurology: Parkinson's disease. New York: Raven Press, pp 45-345

Green J, McDonald WM, Vitek JL, Evatt M, Freeman A, et al. (2002) Cognitive impairments in advanced PD without dementia. Neurology 59: 1320-1324. http:// dx.doi.org/10.1212/01.WNL.0000031426.21683.E2

Hoddes E, Zarcone V, Smythe H, Phillips R, Dement WC (1973) Quantificatication of sleepiness: a new approach. The International Journal of Psychophysiology 10 (4): 431-436. doi: 10.1111/j.1469-8986.1973. tb00801.x

Hoehn MM, Yahr MD (1967) Parkinsonism: onset, progression and mortality. Neurology 17 (5): 427-42. doi: 10.1212/WNL.17.5.427

Hwang C, Yeh C-T, Zhu Q, Pal PK, Calne S, Samii A, Fleming JAE (1999) A review of normal sleep and its disturbances in Parkinson's disease. Parkinsonism and Related Disorders 5: 1-17. http://dx.doi. org/10.1016/S1353-8020(99)00011-5

Iranzo A, Molinuevo JL, Santamaría J, Serradell M, Martí MJ, et al. (2006) Rapideye- movement sleep behaviour disorder as an early marker for a neurodegenerative disorder: a descriptive study.
The Lancet Neurology 5: 572-577. http://dx.doi. org/10.1016/S1474-4422(06)70476-8

Janvin C, Aarsland D, Larsen JP, Hugdahl K (2003) Neuropsychological profile of patients with Parkinson's disease without dementia. Dementia and Geriatric Cognitive Disorder 15: 126-131. doi: $10.1159 / 000068483$

Janvin CC, Larsen JP, Aarsland D, Hugdahl K (2006) Subtypes of mild cognitive impairment in Parkinson's disease: progression to dementia. Movement Disorder 21: 1343-1349. doi: 10.1002/mds.20974

Kostic VS, Susic V, Przedborski S, Sternic N (1991) Sleep EEG in depressed and nondepressed patients with Parkinson's disease. The Journal of Neuropsychiatry and Clinical Neuroscience 3: 176-179

Lavie P, Wajsbort J, Youdim MBH (1980) Deprenyl does not cause insomnia in parkinsonian patients. Communications Psychopharmacology 4 (4): 303-307

Levin BE, Llabre MM, Reisman S, Weiner WJ, SanchezRamos J, et al. (1991) Visuospatial impairment in Parkinson's disease. Neurology 41: 365-369. doi: 10.1212/WNL.41.3.365

Marinus J, Visser M, van Hilten JJ, Lammers GJ, Stiggelbout AM (2003) Assessment of sleep and sleepi $\neg$ ness in Parkinson disease. Sleep 26 (8): 10491054

Marinus J, Visser M, Verwey NA, Verhey FR, Middelkoop HA, et al. (2003) Assessment of cognition in Parkinson's disease. Neurology 61: 12221228. doi: 10.1212/01.WNL.0000091864.39702.1C

Marion M-H, Qurashi M, Marshall G, Foster O (2008) Is REM sleep Behaviour Disorder (RBD) a risk factor of dementia in idiopathic Parkinson's disease? Neurology 255: 192-196. doi: 10.1007/s00415-0080629-9

Massicotte-Marquez J, De'cary A, Gagnon JF, Vendette M, Mathieu A, et al. (2008) Executive dysfunction and memory impairment in idiopathic REM sleep behavior disorder. Neurology 70: 1250-1257

Mehta SH, Morgan JC, Sethi DK (2008) Sleep Disorders Associated wich Parkinson's Disease: role of dopamine, epidemiology, and clinical scale assessment. CNS Spectrums 13: 3 (Suppl 4): 6-11

Menza MA, Rosen RC (1995) Sleep in Parkinson's disease: the role of depression and anxiety. Psychosomatics 36: 262-266. http://dx.doi. org/10.1016/S0033-3182(95)71665-6

Molloy DW, Alemayehu E, Roberts R (1991) Reliability of a Standardized Mini-Mental State Examination compared with the traditional Mini-Mental State Examination. American Journal of Psychiatry 148: 102 $-105$ 
Muslimovic D, Post B, Speelman JD, Schmand B (2005) Cognitive profile of patients with newly diagnosed Parkinson disease. Neurology 65: 1239-1245. doi: 10.1212/01.wnl.0000180516.69442.95

Perfetti B, Varanese S, Mercuri P, Mancino E, Saggino A, Onofrj M (2010) Behavioural assessment of dysexecutive syndrome in Parkinson's disease without dementia: A comparison with other clinical executive tasks. Parkinsonism and Related Disorders 16: 45-60. doi: 10.1016/jparkreldis.2009.07.011

Porter B, Macfarlane R, Walker R (2008) The frequency and nature of sleep disorders in a community-based population of patients with Parkinson's disease. European Jounal of Neurology 15 (1): 50-54. doi: 10.1111/j.1468-1331.2007.01998.x

Razmy A, Lang AE, Shapiro CM (2004) Predictors of impaired daytime sleep and wakefulness in patients with Parkinson disease treated with older (ergot) vs newer (nonergot) dopamine agonists. Archives of Neurology 61 (1): 97-102. doi:10.1001/ archneur.61.1.97

Ribeiro S (2003) Sonho, memória e o reencontro de Freud com o cérebro. Revista Brasileira de Psiquiatria 25 (Supp II): 59-63. http://dx.doi.org/10.1590/S151644462003000600013

Richard IH, Schiffer BB, Kurlan R (1996) Anxiety in Parkinson's disease. The Journal of Neuropsychiatry and Clinical Neurosciences 8 (4): 383-392

Rosa JT, Malandrin MA, Leite EC, Silva JCV (1986) Um estudo sobre a validação do Inventário de Depressão de Beck. Relato de pesquisa. Anais do Congresso da Sociedade Interamericana de Psicologia, Caracas, Venezuela

Rossi C, Volterrani D, Nicoletti V, Manca G, Frosini D (2009) "Parkinson-dementia" diseases: a comparison by double tracer SPECT studies. Parkinsonism and Related Disorders 15: 762-766. doi: 10.1016/j. parkreldis.2009.05.012

Schenck CH, Bundlie SR, Mahowald MW (1996) Delayed emergence of a parkinsonian disorder in 38\% of 29 older men initially diagnosed with idiopathic rapid eye movement sleep behaviour disorder. Neurology 46: 388- 393

Starkstein SE, Preziosi TJ, Robinson RG (1991) Sleep disorders, pain and depression in Parkinson's disease. European Journal of Neurology 31: 352-355. doi: 10.1159/000116692

Stavitsky K, Neargarder S, Bogdanova Y, McNamara P, Cronin-Golomb A (2012) The impact of sleep quality on cognitive functioning in Parkinson's disease. The Journal of the International Neuropsychological Society 18: 108-17. http://dx.doi.org/10.1017/ S1355617711001482
Stevens S, Cormella CL, Stepanski EJ (2004) Daytime sleepiness and alertness in patients with Parkinson disease. Sleep 27 (5): 967-972

Trenkwalder C (1998) Sleep dysfunction in Parkinson's disease. Journal of Clinic Neuroscience 5: 107-14

Troster AI, Stalp L, Paolo A, Fields J, Koller W (1995) Neuropsychological impairment in Parkinson's disease with and without dementia. Archives of Neurology 52: 1164-9. doi: 10.1001/ archneur.1995.00540360042014

Van Hilten B, Hoff JI, Middelkoop HA, van der Velde EA, Kerkhof GA, et al. (1994) Sleep disruption in Parkinson's disease: assessment by continuous activity monitoring. Archives of Neurology 51 (9): 922928. doi: 10.1001/archneur.1994.00540210094018

Vendette M, Gagnon JF, De'cary A, Massicotte-Marquez R.B Postuma RB, et al. (2007) REM sleep behavior disorder predicts cognitive impairment in Parkinson disease without dementia. Neurology 69: 1843-1849. doi: 10.1212/01.wnl.0000278114.14096.74

Wright MJ, Burns RJ, Geffen GM, Geffen LB ( 1990) Covert orientation of visual attention in Parkinson's disease: an impairment in the maintenance of attention. Neuropsychology 28: 151-159. http://dx.doi. org/10.1016/0028-3932(90)90097-8

Zadikoff C, Fox SH, Tang-Wai DF, Thomsen T, de Bie MA, et al. ( 2007) A comparison of the mini mental state exam to the Montreal cognitive assessment in identifying cognitive deficits in Parkinson's disease. Movement Disorder 23 (2): 297-9. doi: 10.1002/ mds. 21837

Zhang L, Dong J, Liu W, Zhang Y, (2013) Subjective poor sleep quality in Chinese patients with Parkinson's disease without dementia. The Journal of Biomedical Research 27 (4): 291-295. doi: 10.7555/ jbr.27.20120143 


\section{Relación entre la calidad del sueño y función cognitiva en pacientes con la enfermedad de Parkinson}

Resumen. Describir las características clínicas de pacientes con la enfermedad de Parkinson, así como la relación entre la calidad del sueño y las funciones cognitivas. Se realizó un estudio descriptivo-analítico de corte transversal que evaluó: los episodios de depresión, ansiedad, estado de salud general, progresión de la enfermedad, sueño, calidad del sueño y funciones cognitivas de 24 pacientes con enfermedad de Parkinson sin demencia. Se observó una relación inversa de tipo moderada entre la memoria tardía y trastornos del sueño. El componente de la latencia del sueño tuvo una correlación inversa moderada con la memoria, y con la función visuoespacial. También se observaron otras relaciones entre la calidad subjetiva del sueño y la función visuoespacial, la latencia del sueño y el resultado global de la SCOPA-COG, y entre la duración del sueño y la atención. Se observó que estas correlaciones fueron inversas y de carácter débil. Este estudio sugiere que los déficits cognitivos leves en ausencia de demencia se relacionaron con la mala calidad del sueño.

Palabras clave: Enfermedad de Parkinson; trastornos del sueño; síntomas cognitivos.

\section{Relaçáo entre qualidade do sono e funçóes cognitivas em pacientes com doença de Parkinson}

Resumo. Descrever as características clínicas e demográficas de pacientes com Doença de Parkinson, bem como verificar a relação entre a qualidade do sono e as funções cognitivas. Trata-se de um estudo descritivo-analítico de corte transversal em que foram avaliados episódios de depressão, ansiedade, estado geral e progressão da doença, sonolência, qualidade do sono e funções cognitivas de 24 pacientes com Doença de Parkinson sem demência. Analisando os resultados do teste de memória tardia e a as alterações do sono, encontrouse correlação do tipo inversa e moderada. $\mathrm{O}$ componente latência do sono teve uma correlação inversa e moderada com a memória, e também com a função visuoespacial. Outras relações também foram observadas como entre a qualidade subjetiva do sono e a função visuoespacial, a latência do sono e o resultado global do SCOPA-COG, e entre a duração do sono e a atenção. Todas essas correlações foram inversas e de caráter fraco. Esse estudo sugere que os déficits cognitivos leves, na ausência de demência, foram relacionados com qualidade ruim do sono.

Palavras-chave: Doença de Parkinson; distúrbio do sono; sintomas cognitivos. 